\title{
Another Way to Implement Diffraction Contrast in SEM
}

\author{
Xiaodong Tao and Alwyn Eades
}

Department of Materials Science and Engineering, Lehigh University, Bethlehem, PA 18015

Diffraction contrast is a challenge for SEM. The ECCI or ECI (Electron Channeling Contrast Imaging) technique successfully used diffraction contrast to image dislocations and other defects in bulk materials[1]. However, ECCI suffers from strict requirements on optics. We are concerned with detecting and imaging dislocations in the SEM using EBSD (Electron Backscatter Diffraction). Our first experiments sought to image the dislocations through a method reciprocal to the ECCI method. This involves measuring the shift of the Kikuchi bands to sub-pixel resolution[2]. However, this method needs complex image processing routines and a long pattern capture time. In this paper, we propose a new method to obtain diffraction contrast, which is still based on EBSD.

The main features in an EBSD pattern, figure 1 and figure 3, are criss-crossed Kikuchi bands. The intensity distribution across a band has the shape of figure 2. As the electron beam scans the crystalline sample, EBSD patterns are captured and then indexed for each point. The automatic indexing of the diffraction pattern in real time, through the Hough transform, handles large orientation differences easily. However, this method is not effective when describing the small orientation differences caused by defects. The strain field of dislocations not only shifts the Kikuchi bands in EBSD patterns, but also changes the intensity distribution of the diffraction bands, as in the case of electron channeling patterns[3]. Thus, the intensity distribution inside or around one or more bands contains information about the dislocations and strain fields. In this research, we have made measurement of the variation of the intensity of the Kikuchi bands and zone axes (through several parameters calculated from the data). Maps of these parameters reveal their potential usefulness as monitors of strain. Instead of doing ordinary Hough Transforms for the diffraction patterns and then indexing them one by one as is done in ordinary EBSD software[4], our program will calculate the intensity distribution of selected bands or zone axes in the EBSD patterns ins tead. In the normalized maps, each pixel corresponds to one EBSD pattern.

As a test, we applied this method to a polycrystalline Ni sample. An FEI XL30 ESEM with a W filament was used. The EBSD system was TSL's OIM system installed with an IMAGRAPH video capture card and a SIT Camera. Figures $4 \mathrm{a}-4 \mathrm{~d}$ are one set of images generated by the above method. In our program, we defined several parameters, such as the mean of the central area of the band divided by the mean of the pattern (figure 4a), and the mean of the zone axis (figure $4 \mathrm{~b}$ ), the mean of the zone axis divided by the mean of the pattern (figure 4c, 4d), and the standard deviation of the band (or zone axis) divided by the standard deviation of the pattern. All of them show good diffraction contrast. These figures can be compared with figure 5, the inverse pole figure. Experiments on characterizing small defects in the semi-conductor sample using this method are still on the way[5,6].

\section{References:}

[1] P.Mortin, M.Pitval, E. Vicario and G.Fontaine, Scanning Vol. 2 217-224 (1979).

[2] Xiaodong Tao and Alwyn Eades, Microscopy and Microanalysis Vol 7, Supp 2 P366.

[3] David C. Joy, Dale E. Newbury and David L. Davidson J. Appl. Phys., vol.53, No.8 R81-R122.

[4] N.C.Krieger Lassen, D.J. Jesson and K. Conradson, Scanning Microscopy, 6 (1992) 115-121.

[5] We are grateful to TSL for allowing us to use their source code.

[6] Support from DOE, under grant DE-FG02-00ER45819, is gratefully acknowledged. 

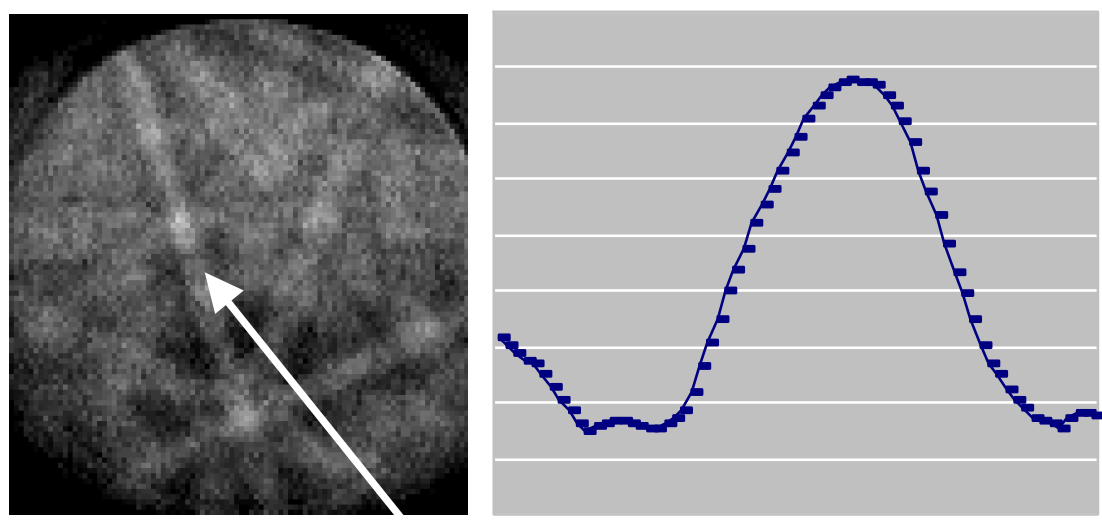

Figure 1. An EBSD pattern from Nickel. The band showed by the arrow head is 100
Figure 2. The averaged intensity distribution across the 100 band in figure 1

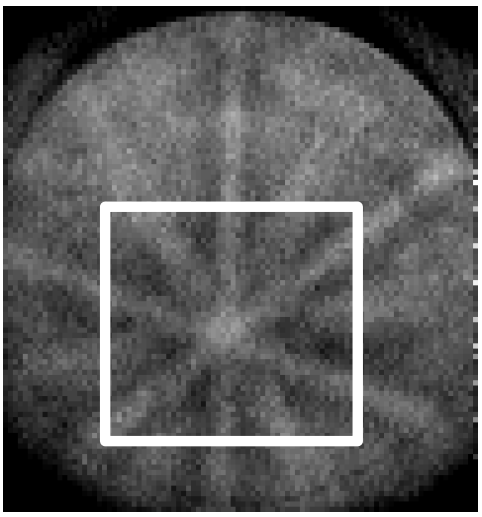

Figure 3. The box marks the region around the $\overline{101}$ zone axis used for the mapping

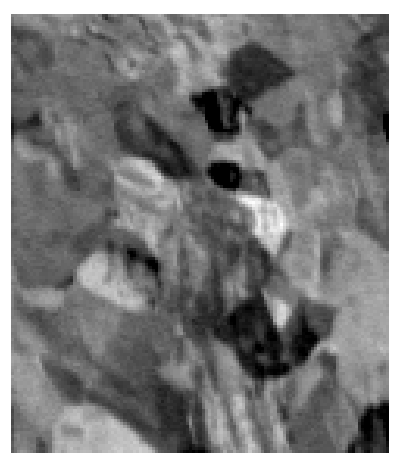

Figure 4a

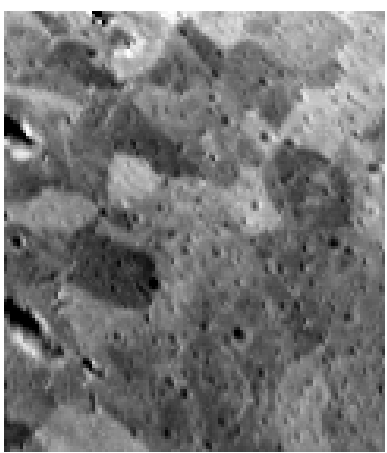

Figure $4 b$

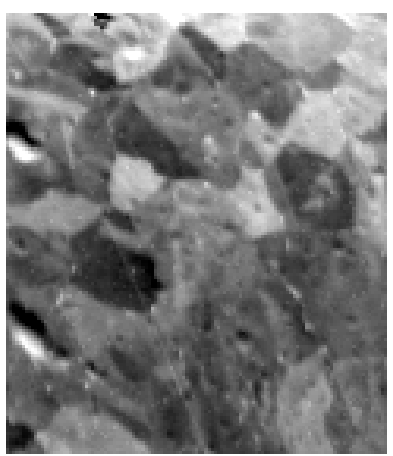

Figure 4c

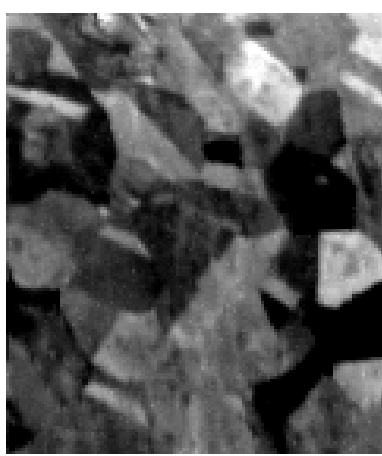

Figure 4d

Figure 4a. Map of the mean of the center area of the100 band divided by the mean of the pattern. Figure 4b. Map of the mean of the 101 zone axis (showed in figure 3). This map has a blend of diffraction contrast and topographic contrast.

Figure 4c. Map formed by the mean of the 101 zone axis divided by the mean of the pattern. Most of the contrast comes from diffraction contrast.

Figure 4d. Map formed by the mean of the 101 zone axis divided by the mean of the pattern.

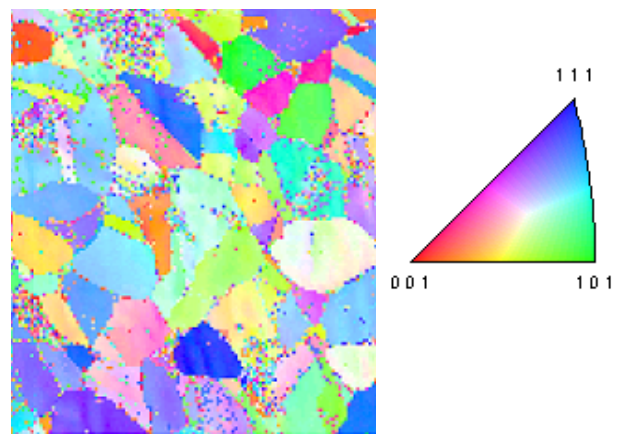

Figure 5. Inverse Pole Figure map of Ni Polycrystalline sample. Compare with figures $4 \mathrm{a}, 4 \mathrm{~b}, 4 \mathrm{c}$, and $4 \mathrm{~d}$. 